\title{
Desarrollo de un Arreglo Circular de Antenas utilizando herramientas de Electromagnetismo Computacional
}

\author{
Celia M. Calderón ${ }^{(1),(2),(3)}$, Héctor M. Pérez ${ }^{(1) *}$, Alberto M. Benavides ${ }^{(2)}$ y Luis J. Morales ${ }^{(2)}$. \\ (1) Instituto Politécnico Nacional, Sección de Estudios de Posgrado e Investigación, Escuela Superior de \\ Ingeniería Mecánica y Eléctrica Unidad Culhuacán, Av. Santa Ana No. 1000, Col. San Francisco \\ Culhuacán, 04430, México D.F. -México ( e-mail: celiacalderon@gmail.com, hmperezm@ipn.mx*) \\ (2) Facultad de Ingeniería en Electrónica y Comunicaciones, Universidad Veracruzana, Av. Venustiano \\ Carranza s/n. Col Revolución, Poza Rica Ver.(e-mail: abenavides@uv.mx, lumorales@uv.mx) \\ (3) Facultad de Ingeniería Mecánica Eléctrica, Universidad Veracruzana. Av. Venustiano Carranza s/n. \\ Col Revolución, Poza Rica Ver. -México
}

${ }^{*}$ Autor a quien debe dirigirse la correspondencia.

Recibido May. 28, 2013; Aceptado Jul. 17, 2013; Versión final recibida Ago. 12, 2013

\begin{abstract}
Resumen
Se presenta el diseño de un arreglo circular de antenas con un difractor de geometría variable colocado en su interior. La idea es disponer de una metodología para la simulación de diversos arreglos circulares de antenas para trabajar en rangos de frecuencias no ionizantes, una limitante de los rayos $\mathrm{X}$ en exámenes tales como las mamografías. El arreglo es modelado utilizando herramientas del electromagnetismo computacional: 1) el método de diferencias finitas en el dominio del tiempo, para transformar las ecuaciones diferenciales de Maxwell en ecuaciones de diferencia finita y resolverlas; 2 ) el método de capas perfectamente acopladas, para establecer las condiciones de frontera de absorción del sistema; y 3) las condiciones de conductor eléctrico perfecto, para modelar el difractor. El algoritmo diseñado asegura que no existen reflexiones espurias que puedan afectar las lecturas de los sensores y el comportamiento electromagnético del sistema. Los resultados muestran que es factible emplear el arreglo de antenas propuesto para el desarrollo de un sistema de detección temprana de cáncer de mama operando con frecuencias no ionizantes.
\end{abstract}

Palabras clave: arreglo circular de antenas, diferencias finitas, capas perfectamente acopladas, electromagnetismo computacional

\section{Development of a Circular Antenna Array using Computational Electromagnetics Tools}

\begin{abstract}
The design of a circular antenna array, with a variable geometry diffracting device placed in it. The idea is to develop a methodology for the simulation of circular antenna array to work in frequency non-ionizing ranges, a limitation of $X$ ray exams such as those for early detection of breast cancer. The array is modeled using computational electromagnetics tools: 1 ) The method of finite differences in time domain, to transform Maxwell differential equations into finite difference equations to solve them. 2) the method of perfectly matched layers, to establish the absorbing boundary conditions of the system and 3) the conditions of perfect electrical conductor to model the diffracting device. The designed algorithm ensures that there are no spurious reflections which could affect the readings of the sensors and the electromagnetic behavior of the system. Results show that the proposed antenna array is suitable for the development of a system for the early detection of breast cancer working at non ionizing frequencies.
\end{abstract}

Keywords: antennas array, finite differences, perfectly matched layer, computational electromagnetics 


\section{INTRODUCCIÓN}

El alto índice de mortalidad por cáncer de mama en todo el mundo ha generado el interés de muchos investigadores para proponer la tecnología de microondas para la detección temprana de este mal. Susan Hagness pionera de esta línea de investigación, propone emigrar al rango de frecuencias no ionizantes (Aponte et al., 2007) para la detección oportuna del cáncer de mama en mujeres menores de 40 años, ya que esta es una limitante de los rayos X del examen de mamografía, la cual opera a frecuencias ionizantes, y debido a la radiación recibida por el cuerpo humano no es permitido realizarse en períodos menores a 6 meses.

Primeramente se requiere diseñar un arreglo de antenas que opere a la frecuencia de microondas, por lo que en este artículo se presentan los resultados del primer arreglo de antenas que desarrollan los autores. Los tipos de arreglos que se han diseñado por diversos grupos de investigadores son arreglos de antenas planares o circulares. En la literatura se hace referencia a los resultados de diversas investigaciones reportados para ser usados para la detección oportuna de cáncer de mama, entre ellos se encuentran: arreglos circulares de 17 antenas, realizado por la pionera en esta investigación (Hagness et.al., 1998; Nilavalan et al., 2003; Converse et al., 2004; Converse et al., 2006); el modelado de un sistema de 10, 15, 30 por (Fear et al., 2002); de 14 antenas presentado por (O'Halloran et al., 2010), casos de 32 antenas, fueron realizados por (Meaney et al., 2000; Fear y Stuchly 2000); de 40 antenas (Winters et al., 2008) y el de 64 antenas por (Henriksson et al., 2009).

Investigaciones referentes a casos de arreglos planares de antenas se tienen: 11 antenas de (Bassi et al., 2012), 17 antenas usadas por (Conceicao et al., 2009) haciendo una comparación entre ambos tipos de arreglos por (Johnson et al., 2009) y de 49 antenas por (Li et al., 2004). Para investigaciones con arreglos dispuestos en forma tridimensional se tienen: 72 antenas por (Xie et al., 2006), de 40 antenas por (Shea et. al., 2010; De Lorenzo et al., 2008); arreglos tanto planares como circulares $5 \times 5$ (Fear et al., 2002; Conceicao et al., 2009); un estudio documentado con un arreglo de cruz que contiene solo dos antenas colocadas en forma perpendicular es el presentado por (Hagness et al., 1999), uno de antena de corneta (Amineh et al., 2011). Todas las investigaciones mencionadas anteriormente se realizan con una aportación común: trabajar en el rango de frecuencias no ionizantes.

El interés principal al realizar esta investigación, inicia con el desarrollo de un arreglo circular de antenas, que será utilizado posteriormente para la construcción de un prototipo que permita detectar tumores basados en las propiedades dieléctricas del tejido maligno, con respecto al tejido sano. Desde el punto de vista electromagnético, los parámetros de conductividad y permitividad eléctrica dependen de la cantidad de agua almacenada en cada zona del tejido mamario. Se requiere sentar las bases para la implementación futura de una gran diversidad de arreglos de antenas, hasta encontrar el arreglo óptimo para la investigación que se desea realizar.

En este artículo, los parámetros eléctricos que definen el comportamiento del tejido mamario (incluyendo los tumores) no se abordan, ya que deseamos centrar nuestra atención en modelar el difractor colocado en el interior del arreglo, para comprobar el buen funcionamiento del arreglo de antenas diseñado primeramente; iniciamos discretizando el área del mismo y estableciendo las condiciones de Conductor Eléctrico Perfecto, discretización que será utilizada como base en la investigación que se desea realizar posteriormente a partir de este algoritmo y aplicarlos al modelado de los tumores.

Referente a las herramientas del electromagnetismo computacional, se utiliza primeramente el Método de Diferencias Finitas en el Dominio del tiempo (FDTD), con la finalidad de transformar las ecuaciones diferenciales de Maxwell y transformarlas en ecuaciones de diferencia finita, resolviendo algebraicamente las ecuaciones electromagnéticas del sistema dividiendo la región de cálculo en celdas pequeñas uniformes (celdas de Yee). Posteriormente se requiere limitar la propagación infinita de la onda electromagnética alimentada por la antena transmisora (Tx) mediante las Condiciones de Frontera de Absorción, el Método utilizado es el de Capas Perfectamente Acopladas (PML). Por último se hace uso de la condiciones de Conductor Eléctrico Perfecto, determinando las ecuaciones del Campo Eléctrico y Magnético para modelar el difractor en el interior del arreglo de antenas.

En este arreglo de antenas se designa como transmisora la primera de las 16 antenas (Tx1) y las 15 restantes como antenas receptoras (sensores o probes: P1 a P15), posteriormente se considera la segunda antena (Tx2) como transmisora y las otras 15 restantes como receptoras, así suscesivamente hasta realizar un barrido en todo el sistema, desde la antena transmisora Tx1 hasta la Tx16, la localización de cada antena debe ser considerada en el algoritmo de programación así también como el intercambio de la posición de las antenas receptoras respectivamente ( $P 1$ a $P 15)$. La señal de alimentación es un pulso Gaussiano diferenciado con frecuencia central de operación de $7.5 \mathrm{GHZ}$ ya que se requiere que trabaje en 
el rango de frecuencias no ionizantes. Se analizan particularmente tres casos de estudio, ya que se modela el difractor con diferentes formas geométricas (cuadrado, circular y elíptico) en el interior del arreglo de antenas, específicamente en el centro, mediante la implementación de las condiciones de conductor eléctrico perfecto.

Al resolver las ecuaciones de Maxwell mediante las herramientas del electromagnetismo computacional utilizadas se realizan las lecturas de los sensores obteniendo: 1) los valores de Campo Eléctrico y Campo Magnético medidos en los sensores o probes; 2) la Potencia Reflejada por el difractor, esto se logra calculando la potencia del sistema sin difractor (Potencia Incidente), posteriormente calculando la potencia del sistema con difractor (Potencia Total), siendo la diferencia entre estas dos potencias, la Potencia Reflejada por el difractor. El algoritmo diseñado se programa en MatLab® (Elsherbeni y Demir, 2009) utilizándolo también como interfaz gráfica.

\section{SISTEMA PROPUESTO}

El sistema propuesto se detalla a continuación exponiendo cada etapa del proceso considerada en el algoritmo diseñado. Las herramientas del Electromagnetismo Computacional que permiten diseñar la región de cálculo en donde se simula el arreglo de antenas con el difractor en su interior son:

\section{Método de Diferencias Finitas en el Dominio del tiempo (FDTD)}

Durante los pasados 25 años el Método de Diferencias Finitas en el Dominio del Tiempo (FDTD, por sus siglas en inglés) (Taflove y Hagness, 2005; Yu et al., 2006) es una de las herramientas del electromagnetismo computacional que se utiliza para resolver las ecuaciones de Maxwell en el dominio del tiempo. El método debe su existencia al poder de la simplicidad que proporciona, por lo que es considerado uno de los más sencillos, donde se transforman las ecuaciones diferenciales parciales en ecuaciones de diferencia finita, que son aproximaciones de forma algebraica y relacionan el valor de la variable dependiente en un punto en la región de solución a los valores en algunos puntos cercanos.

Los valores que representan este caso de estudio se muestran en la Figura 1, donde se muestra la región de cálculo que consiste en $1300 \times 1300$ celdas de Yee, en los ejes X y Y, respectivamente, siendo un total 1 '690,000 celdas.

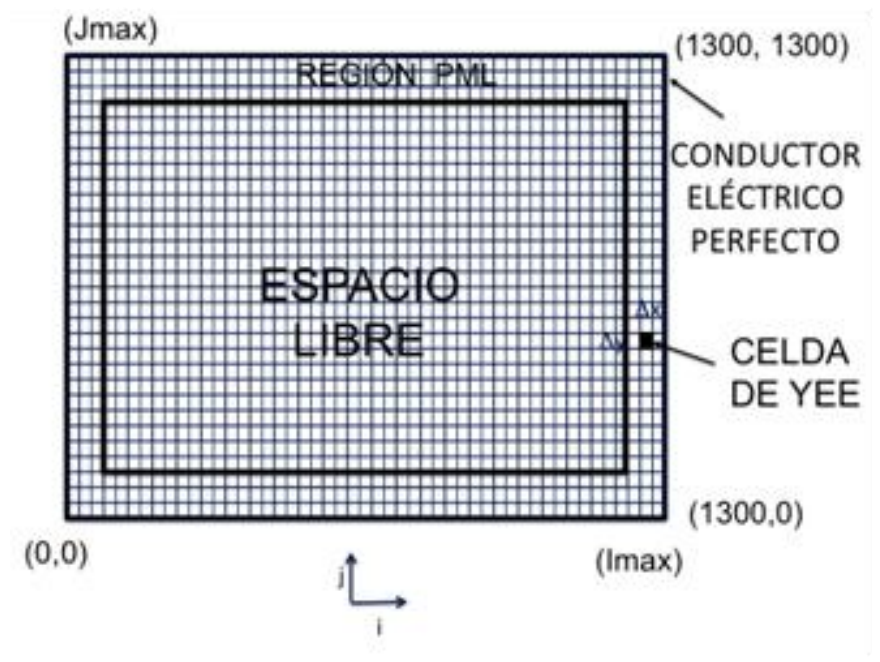

Fig. 1: Representación de la región de cálculo

\section{Método de Capas Perfectamente Acopladas (PML)}

Las condiciones de frontera de absorción se utilizan para truncar la propagación infinita de una onda electromagnética, de tal forma que al llegar a la frontera de la región de cálculo la onda propagada sufra una atenuación hasta ser completamente "absorbida", el método más utilizado es el Método de Capas Perfectamente Acopladas PML (por sus siglas en inglés), (Berenger, 1994; Sullivan, 2000; Benavides et al., 2009). Actualmente se cuenta con una modificación de este método, propuesto por (Benavides et al., 2010) como una versión modificada del PML y es el que se utiliza en el presente trabajo ya que proporciona la reducción de las ecuaciones que se utilizan para la implementación del método propuesto. 
Partiendo del conjunto de ecuaciones de Maxwell que representan las condiciones para la simulación del sistema tipo Transversal Magnético y Bidimensional (Benavides et al., 2011) utilizado en este artículo son:

$$
\begin{aligned}
& \frac{\partial}{\partial t} H_{z}=-\frac{1}{\mu}\left(\frac{\partial}{\partial x} E_{y}-\frac{\partial}{\partial x} E_{x}\right)-\frac{\sigma^{*}}{\mu} H_{z} \\
& \frac{\partial}{\partial t} E_{x}=\frac{1}{\varepsilon}\left(\frac{\partial}{\partial y} H_{z}\right)-\frac{1}{\varepsilon} J_{s x}-\frac{\sigma}{\varepsilon} E_{x} \\
& \frac{\partial}{\partial t} E_{y}=-\frac{1}{\varepsilon}\left(\frac{\partial}{\partial x} H_{z}\right)-\frac{1}{\varepsilon} J_{s y}-\frac{\sigma}{\varepsilon} E_{y}
\end{aligned}
$$

Donde $\boldsymbol{E}$ es la intensidad de Campo Eléctrico $(\mathrm{V} / \mathrm{m}), H$ es la intensidad de Campo Magnético $(\mathrm{A} / \mathrm{m}), \mu$ es la permeabilidad del espacio libre $(\mathrm{H} / \mathrm{m}), \varepsilon$ denota la permitividad del espacio libre $(\mathrm{F} / \mathrm{m}), \sigma$ es la conductividad eléctrica $(\mathrm{S} / \mathrm{m}), \sigma^{*}$ denota la pérdida magnética equivalente $(\Omega / \mathrm{m})$ y $J$ es la densidad de corriente eléctrica $\left(A / \mathrm{m}^{2}\right)$. Seguidamente, para la implementación de las ecuaciones $\mathrm{PML}$, la componente transversal se descompone en sus proyecciones $X$ y $Y$, por lo que la componente $H_{z}=H_{z x}+H_{z y}$, la conductividad $\sigma$ se indica precisamente a la dirección de propagación. La densidad de corriente $J_{s z}$ se elimina, ya que no existen cargas libres en el medio PML. Por lo que se obtiene el conjunto de cuatro ecuaciones PML siguientes:

$$
\begin{aligned}
& \frac{\partial}{\partial t} E_{y}=-\frac{1}{\varepsilon}\left(\frac{\partial}{\partial x} H_{z}\right)-\frac{1}{\varepsilon} J_{s y}-\frac{\sigma}{\varepsilon} E_{y} \\
& \frac{\partial}{\partial t} H_{z y}=-\frac{1}{\mu}\left(\frac{\partial}{\partial x} E_{y}-\frac{\partial}{\partial x} E_{x}\right)-\frac{\sigma_{y}^{*}}{\mu} H_{z y} \\
& \frac{\partial}{\partial t} E_{y}=-\frac{1}{\varepsilon}\left(\frac{\partial}{\partial x}\left(H_{z x}+H_{z y}\right)\right)-\frac{\sigma_{x}}{\varepsilon} E_{y} \\
& \frac{\partial}{\partial t} E_{x}=\frac{1}{\varepsilon}\left(\frac{\partial}{\partial x}\left(H_{z x}+H_{z y}\right)\right)-\frac{\sigma_{y}}{\varepsilon} E_{x}
\end{aligned}
$$

Las ecuaciones que definen este sistema e implementadas en el algoritmo, son considerando las condiciones de Conductor Eléctrico Perfecto, en donde el Campo Eléctrico tangencial y el Campo Magnético normal a la superficie del conductor deben ser igual a cero son:

$$
\begin{aligned}
& H z x_{(1: \operatorname{Im} a x, 1: J \max )}=\frac{\left(1-\frac{\sigma_{x}^{*} \Delta t}{2 \mu}\right)}{\left(1+\frac{\sigma_{x}^{*} \Delta t}{2 \mu}\right)} H z x_{(1: \operatorname{Im} a x, 1: J \max )}-\frac{\Delta t}{\mu\left(1+\frac{\sigma_{x}^{*} \Delta t}{2 \mu}\right)}\left[\frac{E y_{(2: \operatorname{Im} a x+1,1: J \max )}-E y_{(1: \operatorname{Im} a x, 1: J \max )}}{\Delta x}\right] \\
& H z y_{(1: \operatorname{Im} a x, 1: J \max )}=\frac{\left(1-\frac{\sigma_{y}^{*} \Delta t}{2 \mu}\right)}{\left(1+\frac{\sigma_{y}^{*} \Delta t}{2 \mu}\right)} H z y_{(1: \operatorname{Im} a x, 1: J \max )}-\frac{\Delta t}{\mu\left(1+\frac{\sigma_{y}^{*} \Delta t}{2 \mu}\right)}\left[\frac{E x_{(1: \operatorname{Im} a x, 2: J \max +1)}-E x_{(1: \operatorname{Im} a x, 2: J \max )}}{\Delta y}\right]
\end{aligned}
$$


$E x_{(1: \operatorname{Im} a x, 1: J \max )}=E x_{(1: \operatorname{lm} a x, 1: J \max )} \frac{\left(1-\frac{\sigma_{y} \Delta t}{2 \varepsilon}\right)}{\left(1+\frac{\sigma_{y} \Delta t}{2 \varepsilon}\right)}+\frac{\Delta t}{\varepsilon\left(1+\frac{\sigma_{y} \Delta t}{2 \varepsilon}\right)}\left[\frac{(H z x+H z y)_{(1: \operatorname{lm} a x, 1: J \max )}-(H z x+H z y)_{(1: \operatorname{Im} a x, 1: J \max -1)}}{\Delta y}\right]$

$E y_{(1: \operatorname{Im} a x, 1: J \max )}=E y_{(1: \operatorname{lm} a x, 1: J \max )} \frac{\left(1-\frac{\sigma_{x} \Delta t}{2 \varepsilon}\right)}{\left(1+\frac{\sigma_{x} \Delta t}{2 \varepsilon}\right)}+\frac{\Delta t}{\varepsilon\left(1+\frac{\sigma_{x} \Delta t}{2 \varepsilon}\right)}\left[\frac{(H z x+H z y)_{(1: \operatorname{lm} a x, 1: J \max )}-(H z x+H z y)_{(-1: \operatorname{lm} a x, 1: J \max )}}{\Delta x}\right]$

En la Figura 2 se muestra la sección del diagrama de flujo general para calcular las componentes de Campo Eléctrico y Magnético, requeridos para poder calcular la Potencia Reflejada por el dieléctrico, en los puntos donde son colocados los sensores del sistema.

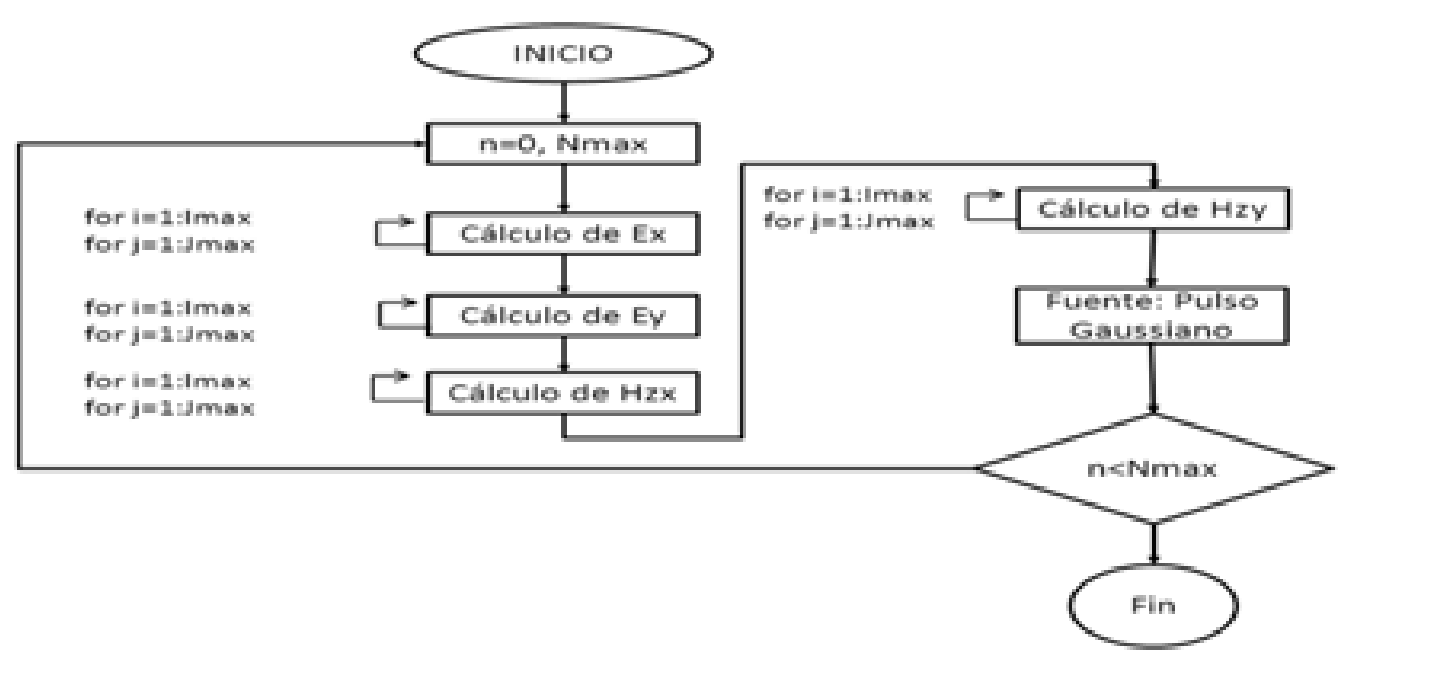

Fig. 2: Diagrama de Flujo del método FDTD

Se considera que el Campo Total es igual al Campo Incidente más el Campo Reflejado, por tanto se calcula los valores de Campo Reflejado mediante la diferencia del Campo Total menos el Campo Incidente, ya que son el interés particular del presente trabajo, esto se obtiene mediante la adecuación de las ecuaciones anteriores para cada tipo de campo en particular, es decir tanto para el Campo Eléctrico, Magnético y la Potencia, mostrado a continuación:

Componentes de Campo Eléctrico Reflejado:

$E_{x r}=E_{x t}-E_{x i}$

$E_{y r}=E_{y t}-E_{y i}$

Seguidamente, las componentes de Campo Magnético Total, Incidente y Reflejado están dados por:

$H_{z t}=H_{z x t}+H_{z y t}$

$H_{z i}=H_{z x i}+H_{z y i}$

$H_{z r}=H_{z t}-H_{z i}$ 
Así la componente de la Potencia Total está dada por:

$P_{x t}=E_{y t} H_{z t}$

$P_{y t}=-E_{x t} H_{z t}$

De los resultados anteriores, la Potencia Total-Total, estará dada por:

$P_{t t}=\sqrt{P_{x t}^{2}+P_{y t}^{2}}$

Componentes de la Potencia Incidente

$P_{x i}=E_{y i} H_{z i}$

$P_{y i}=-E_{x i} H_{z i}$

Seguidamente la Potencia Total Incidente y las componentes de la Potencia Reflejada están dados por:

$P_{t t}=\sqrt{P_{x i}^{2}+P_{y i}^{2}}$

$P_{x r}=P_{x t}-P_{x i}$

$P_{y r}=P_{y t}-P_{y i}$

Potencia Total Reflejada:

$P_{t r}=P_{t t}-P_{t i}$

Todos los valores de Potencia con unidades en Watts. El sistema propuesto trabaja con los parámetros siguientes:

$\lambda=\frac{c}{f}=\frac{3 \times 10^{8}}{7.5 \times 10^{9}}=0,04 \mathrm{~m}$

$\Delta=\frac{\lambda}{80}=0,0005 m$

$\Delta_{t}=\frac{\Delta}{2 c_{0}}=\frac{0,0005}{2\left(3 \times 10^{8}\right)}=8.33 \times 10^{-13} \mathrm{seg}$

Donde $\lambda$ es la longitud de onda $(\mathrm{m}), f$ es la frecuencia de operación $(\mathrm{GHz}), c_{0}$ es la velocidad de la luz $(\mathrm{m} / \mathrm{s}), \Delta$ es el lado de la celda de Yee $(\mathrm{m}), \Delta_{t}$ el escalón temporal (seg) y $T$ es el periodo definido por:

$T=\frac{1}{f_{0}}=\frac{1}{7.5 \times 10^{9}}=1.33 \times 10^{-10} \mathrm{seg}$

\section{Arreglo Circular de 16 Antenas}

Se dispone un arreglo de antenas monopolo, colocadas en forma circular con un diámetro de $20 \mathrm{~cm}$ equivalente a 400 celdas de Yee, la región total incluyendo el PML es de 1300 celdas, la separación entre las antenas es de 22.5, el difractor está colocado en el centro con coordenadas $\mathrm{I}=650, \mathrm{~J}=650$, con un diámetro de 20 celdas, se prueban tres formas geométricas: cuadrado, circular y elíptico. La antena transmisora Tx1, es un monopolo (Allen et al., 2007) que alimenta un pulso gaussiano con frecuencia de operación de $7.5 \mathrm{GHz}$, esta se coloca en las celdas $\mathrm{I}=650, \mathrm{~J}=450$; las 15 antenas receptoras se consideran como los sensores del sistema (probes P1 al P15). En la Figura 3, se muestra el sistema para el caso de un difractor cuadrado. 


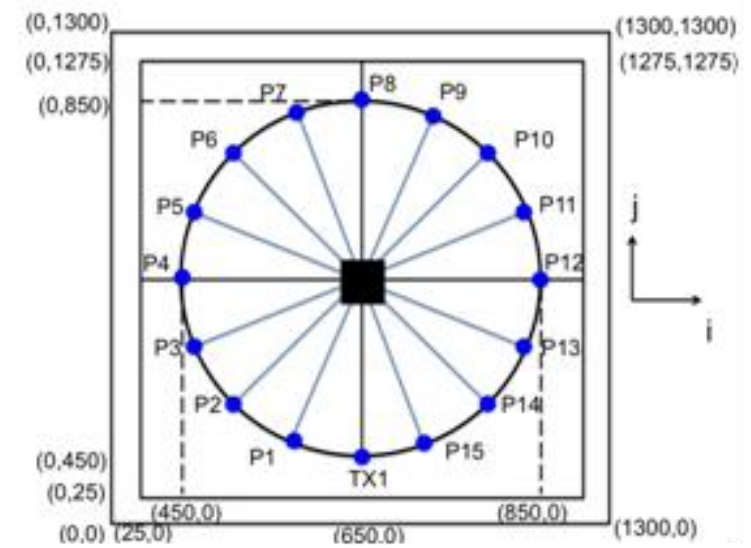

Fig. 3: Arreglo circular de antenas con difractor cuadrado en su interior.

Posteriormente, se considera la antena Tx2 como antena transmisora y las restantes como antenas receptoras (sensores o probes desde P1 a P15), así sucesivamente, considerando la rotación de las antenas Txi siendo $i=1$ a $i=16$, ya que el sistema trabaja haciendo un barrido en el sentido contrario de las manecillas del reloj. Se requiere diseñar 16 plantillas de ubicación de Tx y de los probes P1 al P15 para cada caso, como se muestra en la Tabla 1.

Tabla 1: Plantillas de ubicación para el Arreglo de las 16 Antenas

\begin{tabular}{|c|c|c|c|c|c|c|c|c|c|c|c|c|c|c|c|c|}
\hline Plantilla & \multicolumn{16}{|c|}{ Asignación de la Antena Transmisora (Tx1 a Tx16) y los Sensores o Probes(P1 a P15) } \\
\hline 1 & Tx1 & P2 & P3 & P4 & P5 & P6 & P7 & P8 & P9 & P10 & P11 & P12 & P13 & P14 & P15 & P16 \\
\hline 2 & $\mathrm{P} 1$ & Tx2 & P3 & $\overline{P 4}$ & P5 & P6 & P7 & P8 & P9 & P10 & P11 & P12 & P13 & P14 & P15 & $\mathrm{P} 16$ \\
\hline 3 & $\mathrm{P} 1$ & P2 & $\mathrm{T} \times 3$ & P4 & P5 & P6 & P7 & P8 & P9 & P10 & P11 & P12 & P13 & P14 & P15 & P16 \\
\hline 4 & P1 & P2 & P3 & $\mathrm{T} \times 4$ & P5 & P6 & P7 & P8 & P9 & P10 & P11 & P12 & P13 & P14 & P15 & P16 \\
\hline 5 & $\mathrm{P} 1$ & P2 & P3 & $\mathrm{P} 4$ & Tx5 & P6 & P7 & P8 & P9 & P10 & P11 & P12 & P13 & P14 & P15 & P16 \\
\hline 6 & P1 & P2 & P3 & P4 & P5 & Tx6 & P7 & P8 & P9 & P10 & P11 & P12 & P13 & P14 & P15 & P16 \\
\hline 7 & P1 & P2 & P3 & P4 & P5 & P6 & Tx7 & P8 & P9 & P10 & P11 & P12 & P13 & P14 & P15 & P16 \\
\hline 8 & P1 & P2 & P3 & P4 & P5 & P6 & P7 & Tx8 & P9 & P10 & P11 & P12 & P13 & P14 & P15 & P16 \\
\hline 9 & P1 & P2 & P3 & P4 & P5 & P6 & P7 & P8 & Tx9 & P10 & P11 & P12 & P13 & P14 & P15 & P16 \\
\hline 10 & P1 & P2 & P3 & P4 & P5 & P6 & P7 & P8 & P9 & $T \times 10$ & P11 & P12 & P13 & P14 & P15 & P16 \\
\hline 11 & P1 & P2 & P3 & P4 & P5 & P6 & P7 & P8 & P9 & P10 & Tx11 & P12 & P13 & P14 & P15 & P16 \\
\hline 12 & P1 & P2 & P3 & P4 & P5 & P6 & P7 & P8 & P9 & P10 & P11 & Tx12 & P13 & P14 & P15 & P16 \\
\hline 13 & P1 & P2 & P3 & P4 & P5 & P6 & P7 & P8 & P9 & P10 & P11 & P12 & Tx13 & P14 & P15 & P16 \\
\hline 14 & P1 & P2 & P3 & P4 & P5 & P6 & P7 & P8 & P9 & P10 & P11 & P12 & P13 & $\mathrm{Tx} 14$ & P15 & P16 \\
\hline 15 & P1 & P2 & P3 & P4 & P5 & P6 & P7 & P8 & P9 & P10 & P11 & P12 & P13 & P14 & Tx15 & P16 \\
\hline 16 & P1 & P2 & P3 & P4 & P5 & P6 & P7 & P8 & P9 & P10 & P11 & P12 & P13 & P14 & P15 & Tx16 \\
\hline
\end{tabular}

En la Tabla 2, se muestran las coordenadas de ubicación para formar el arreglo de antenas, con una variación de la posición de la antena transmisora Txi $(i=1, . .16)$, las celdas de Yee correspondientes se representan en function de I y J.

\section{Condiciones de Conductor Eléctrico Perfecto}

Se utilizan estas condiciones para el modelado de cada uno de los difractores, en donde el Campo Eléctrico tangencial y el Campo Magnético transversal son iguales a cero. La discretización para el caso del difractor cuadrado se muestra en la Figura 4 de acuerdo a sus coordenadas de ubicación.

Tabla 2: Coordenadas de ubicación de las Antenas Tx (Celdas de Yee)

\begin{tabular}{|c|c|c|c|c|c|c|c|c|c|c|c|c|c|c|c|c|}
\hline & \multicolumn{16}{|c|}{ Coordenadas $(\mathrm{I}, \mathrm{J})$ para cada antena transmisora $(\mathrm{Tx})$} \\
\hline & $\mathrm{Tx} 1$ & $T \times 2$ & $\mathrm{~T} \times 3$ & $T \times 4$ & Tx5 & Tx6 & $\mathrm{Tx} 7$ & $T \times 8$ & Tx9 & $T \times 10$ & $\mathrm{~T} \times 11$ & Tx12 & $T \times 13$ & Tx14 & Tx15 & $\mathrm{T} \times 16$ \\
\hline $\mathrm{I}=$ & 650 & 573 & 509 & 465 & 450 & 509 & 509 & 573 & 650 & 727 & 791 & 850 & 850 & 835 & 791 & 727 \\
\hline$J=$ & 450 & 465 & 509 & 573 & 650 & 791 & 791 & 835 & 850 & 835 & 791 & 650 & 650 & 573 & 509 & 465 \\
\hline
\end{tabular}




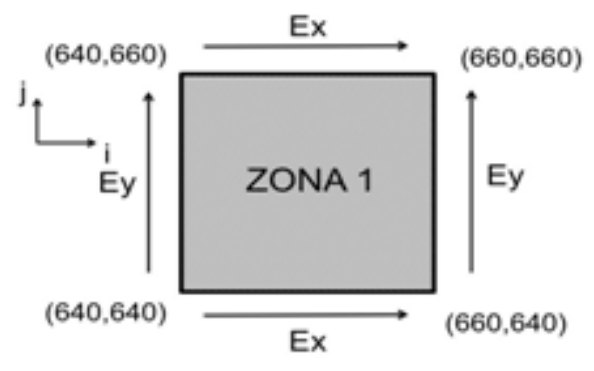

Fig. 4: Zona de Discretización para el difractor cuadrado.

Las condiciones de Conductor Eléctrico Perfecto específicas para el difractor cuadrado en función de la Figura 4 se presentan en la Tabla 3, éstas son incluidas en el algoritmo del sistema. Para el difractor circular se procede de forma semejante para establecer las condiciones PEC, representadas en la Figura 5.

Tabla 3: Condiciones de Conductor Eléctrico Perfecto (PEC) para el difractor cuadrado.

\begin{tabular}{|c|c|c|}
\hline $\begin{array}{c}\text { Zonas del PEC } \\
\text { cuadrado }\end{array}$ & $\begin{array}{c}\text { Componentes Ex } \\
\text { de campo eléctrico }\end{array}$ & $\begin{array}{c}\text { Componentes Ey } \\
\text { de campo eléctrico }\end{array}$ \\
\hline 1 & $\begin{array}{c}\text { Ext }(640: 660,640)=0 ; \\
\text { Ext }(640: 660,660)=0 ;\end{array}$ & $\begin{array}{c}\text { Eyt }(640,640: 660)=0 ; \\
\text { Eyt }(660,640: 660)=0 ;\end{array}$ \\
\hline
\end{tabular}

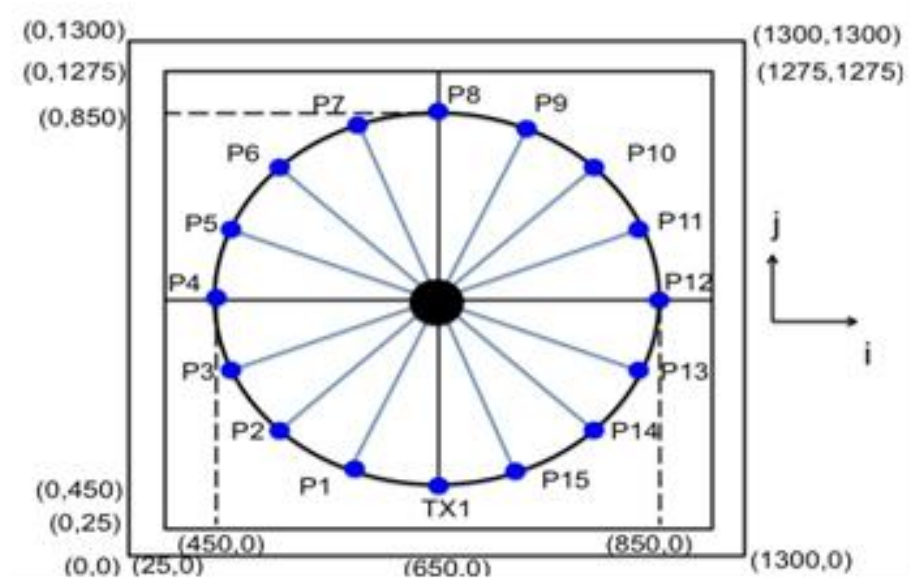

Fig. 5: Arreglo circular de antenas con difractor circular en su interior.

La diferencia con respecto al caso del difractor cuadrado radica en que para modelar el dieléctrico circular la discretización se realiza asignando áreas de formas geométricas que formen el círculo, las 13 zonas consideradas para este caso se representan en la Figura 6.

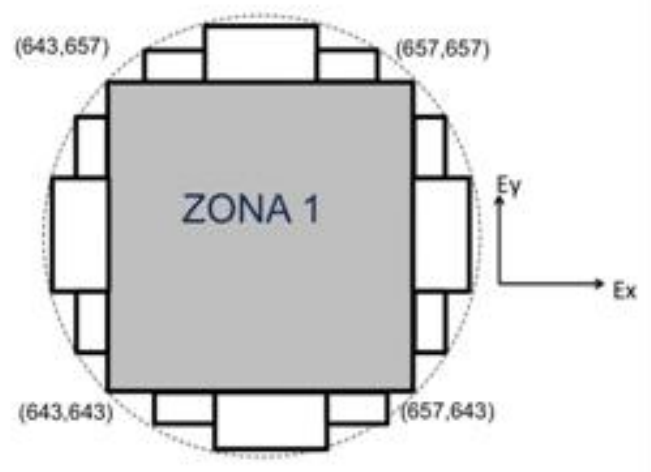

Fig. 6: Zonas de Discretización para el difractor circular. 
Ahora bién, para cada una de las 13 zonas discretizadas se establecen las condiciones PEC, como se muestra en la Tabla 4. El tercer caso de estudio es el difractor elíptico, mostrado en la Figura 7.

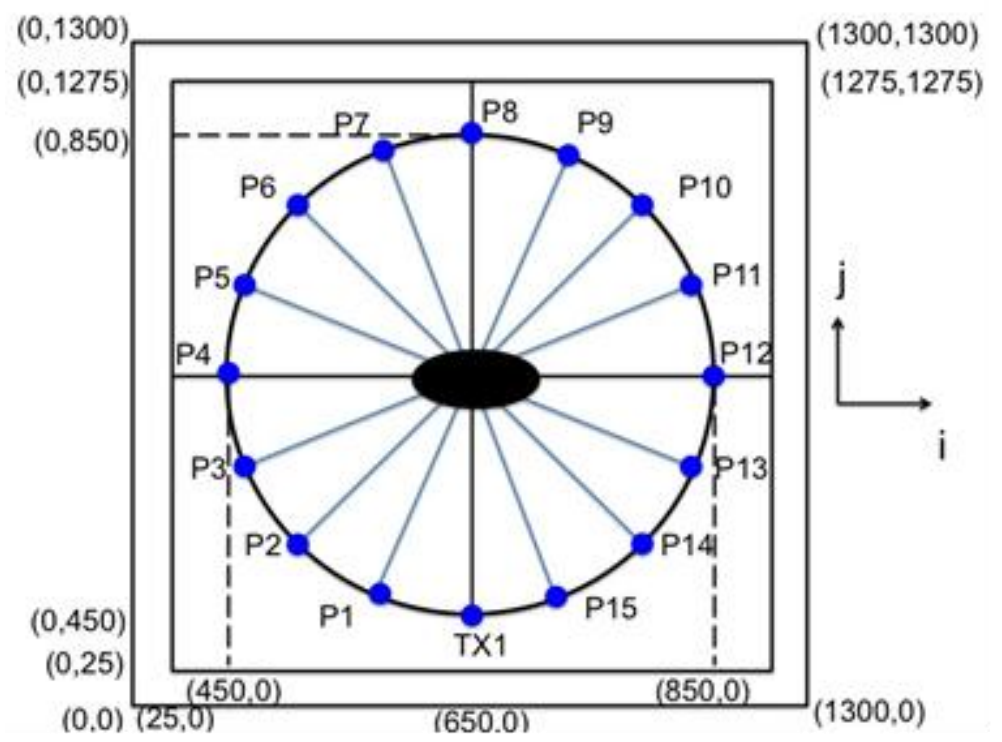

Fig. 7: Arreglo circular de antenas con difractor elíptico en su interior.

Tabla 4: Zonas de Discretización y condiciones PEC para el difractor circular.

\begin{tabular}{|c|c|c|}
\hline Zonas & Componentes Ext & Componentes Eyt \\
\hline 1 & $\operatorname{Ext}(643: 657,643)=0 ; \operatorname{Ext}(643: 657,657)=0$ & $\operatorname{Eyt}(643,643: 657)=0 ; \operatorname{Eyt}(657,643: 657)=0$ \\
\hline 2 & $\operatorname{Ext}(640: 641,647)=0 ; \operatorname{Ext}(640: 641,653)=0$ & $\operatorname{Eyt}(640,647: 653)=0 ; \operatorname{Eyt}(641,647: 653)=0$ \\
\hline 3 & $\operatorname{Ext}(659: 660,647)=0 ; \operatorname{Ext}(659: 660,653)=0 ;$ & $\operatorname{Eyt}(659,647: 653)=0 ; \operatorname{Eyt}(660,647: 663)=0$ \\
\hline 4 & $\operatorname{Ext}(647: 653,640)=0 ; \operatorname{Ext}(647: 653,641)=0$ & $\operatorname{Eyt}(647,640: 641)=0 ; \operatorname{Eyt}(653,640: 641)=0$ \\
\hline 5 & $\operatorname{Ext}(647: 653,659)=0 ; \operatorname{Ext}(647: 653,660)=0$ & $\operatorname{Eyt}(647,659: 660)=0 ; \operatorname{Eyt}(653,659: 660)=0$ \\
\hline 6 & $\operatorname{Ext}(641: 642,645)=0 ; \operatorname{Ext}(641: 642,655)=0$ & $\operatorname{Eyt}(641,645: 655)=0 ; \operatorname{Eyt}(642,645: 655)=0$ \\
\hline 7 & $\operatorname{Ext}(658: 659,645)=0 ; \operatorname{Ext}(658: 659,655)=0$ & $\operatorname{Eyt}(658,645: 655)=0 ; \operatorname{Eyt}(659,645: 655)=0$ \\
\hline 8 & $\operatorname{Ext}(645: 655,641)=0 ; \operatorname{Ext}(645: 655,642)=0$ & $\operatorname{Eyt}(645,641: 642)=0 ; \operatorname{Eyt}(655,641: 642)=0$ \\
\hline 9 & $\operatorname{Ext}(645: 655,658)=0 ; \operatorname{Ext}(645: 655,659)=0$ & $\operatorname{Eyt}(645,658: 649)=0 ; \operatorname{Eyt}(655,658: 659)=0$ \\
\hline 10 & $\operatorname{Ext}(642: 643,644)=0 ; \operatorname{Ext}(642: 643,656)=0$ & $\operatorname{Eyt}(642,644: 656)=0 ; \operatorname{Eyt}(643,644: 656)=0$ \\
\hline 11 & $\operatorname{Ext}(657: 658,644)=0 ; \operatorname{Ext}(657: 658,646)=0$ & $\operatorname{Eyt}(657,644: 656)=0 ; \operatorname{Eyt}(658,644: 656)=0$ \\
\hline 12 & $\operatorname{Ext}(644: 656,642)=0 ; \operatorname{Ext}(644: 656,643)=0$ & $\operatorname{Eyt}(644,642: 643)=0 ; \operatorname{Eyt}(656,642: 643)=0$ \\
\hline 13 & $\operatorname{Ext}(644: 656,657)=0 ; \operatorname{Ext}(644: 656,658)=0$ & $\operatorname{Eyt}(644,657: 658)=0 ; \operatorname{Eyt}(656,657: 658)=0$ \\
\hline
\end{tabular}

La discretización de este difractor elíptico se representa en la Fig. 8, en donde se pueden apreciar 11 zonas diferentes, la Tabla 5 muestra las condiciones de Conductor Eléctrico Perfecto para cada una de las zonas.

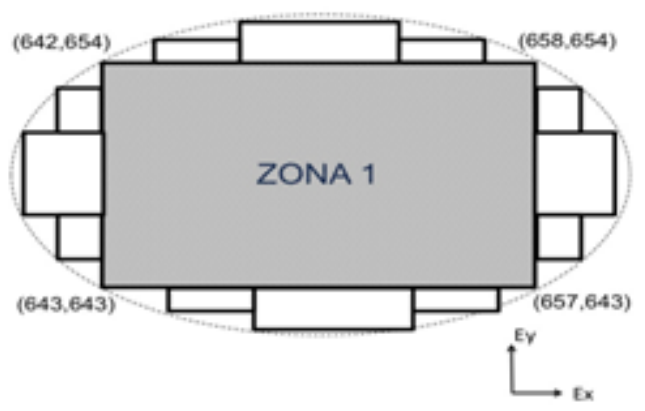

Fig. 8: Zonas de Discretización para el difractor elíptico 
Tabla 5: Zonas de Discretización y condiciones PEC para el difractor elíptico.

\begin{tabular}{|c|c|c|}
\hline Zonas & Componentes Ex & Componentes Ey \\
\hline 1 & $\operatorname{Ext}(642: 658,646)=0 ; \operatorname{Ext}(642: 658,654)=0$ & $\operatorname{Eyt}(642,646: 654)=0 ; \operatorname{Eyt}(658,646: 654)=0$ \\
\hline 2 & $\operatorname{Ext}(640: 641,648)=0 ; \operatorname{Ext}(640: 641,652)=0$ & $\operatorname{Eyt}(640,648: 652)=0 ; \operatorname{Eyt}(641,648: 652)=0$ \\
\hline 3 & $\operatorname{Ext}(641: 642,647)=0 ; \operatorname{Ext}(641: 642,653)=0$ & $\operatorname{Eyt}(641,647: 653)=0 ; \operatorname{Eyt}(642,647: 653)=0$ \\
\hline 4 & $\operatorname{Ext}(659: 660,648)=0 ; \operatorname{Ext}(659: 660,652)=0$ & $\operatorname{Eyt}(659,648: 652)=0 ; \operatorname{Eyt}(660,648: 642)=0$ \\
\hline 5 & $\operatorname{Ext}(658: 659,647)=0 ; \operatorname{Ext}(658: 659,653)=0$ & $\operatorname{Eyt}(658,647: 653)=0 ; \operatorname{Eyt}(659,647: 653)=0$ \\
\hline 6 & $\operatorname{Ext}(643: 657,645)=0 ; \operatorname{Ext}(643: 657,645)=0$ & $\operatorname{Eyt}(643,645: 646)=0 ; \operatorname{Eyt}(657,645: 646)=0$ \\
\hline 7 & $\operatorname{Ext}(645: 655,644)=0 ; \operatorname{Ext}(645: 655,645)=0$ & $\operatorname{Eyt}(645,644: 645)=0 ; \operatorname{Eyt}(655,644: 645)=0$ \\
\hline 8 & $\operatorname{Ext}(648: 652,643)=0 ; \operatorname{Ext}(648: 652,644)=0$ & $\operatorname{Eyt}(648,643: 644)=0 ; \operatorname{Eyt}(652,643: 644)=0$ \\
\hline 9 & $\operatorname{Ext}(643: 657,654)=0 ; \operatorname{Ext}(643: 657,655)=0$ & $\operatorname{Eyt}(643,654: 655)=0 ; \operatorname{Eyt}(657,654: 655)=0$ \\
\hline 10 & $\operatorname{Ext}(645: 655,655)=0 ; \operatorname{Ext}(645: 655,656)=0$ & $\operatorname{Eyt}(646,655: 656)=0 ; \operatorname{Eyt}(655,655: 656)=0$ \\
\hline 11 & $\operatorname{Ext}(648: 652,656)=0 ; \operatorname{Ext}(648: 652,657)=0$ & $\operatorname{Eyt}(648,656: 657)=0 ; \operatorname{Eyt}(652,656: 657)=0$ \\
\hline
\end{tabular}

\section{RESULTADOS EXPERIMENTALES}

Se comprueba la robustez del algoritmo de dos diferentes maneras, una de ellas es verificar que no existen reflexiones espurias en la región PML tal como se muestra en la Figura 9, en donde la propagación de la onda electromagnética aborda la periferia de la región de cálculo sin presentarse reflexiones, es decir, que logra ser debidamente atenuada por la capa PML, esto garantiza que las lecturas de los sensores sean correctas.

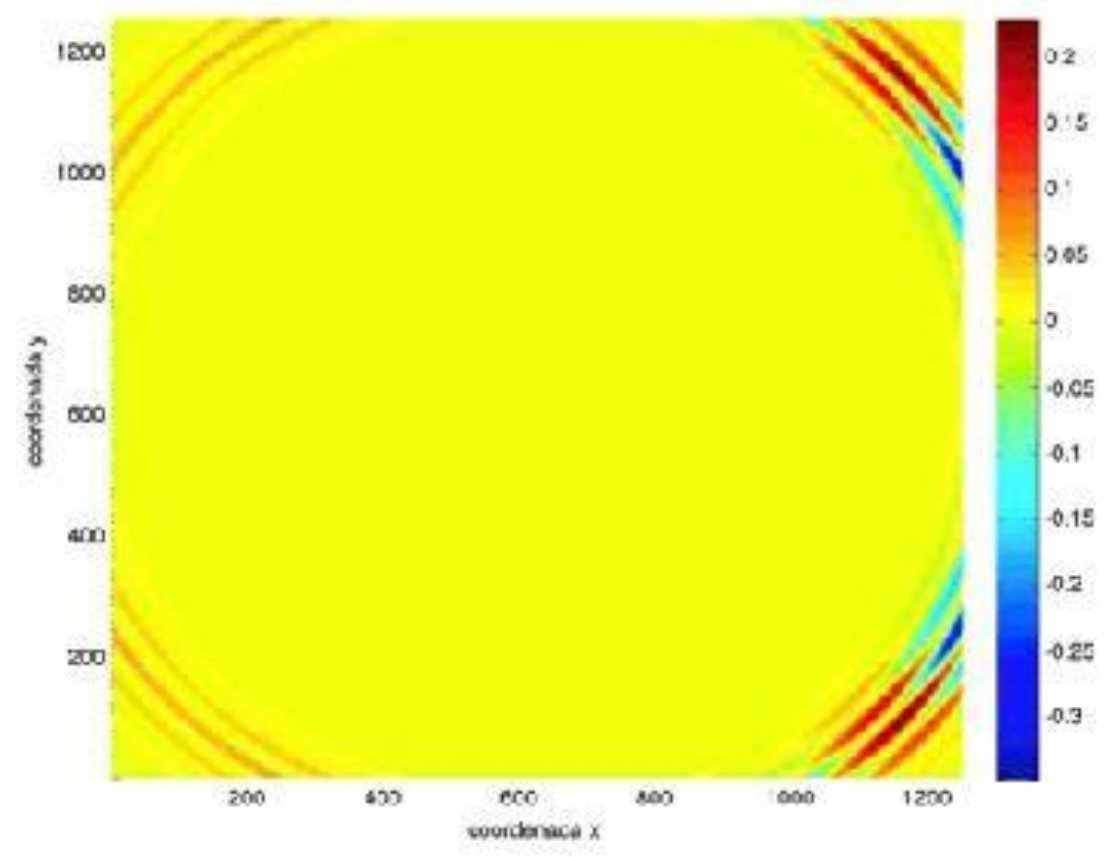

Fig. 9: Campo electromagnético atenuado en la periferia de la región PML.

De acuerdo a los resultados se obtienen los valores de la componente de Campo Eléctrico en un total de 2500 iteraciones para cada uno de los 3 casos de estudio, notándose como la propagación de este campo incide sobre el difractor y continúa su recorrido hasta llegar a la periferia de la región de cálculo.

En las Figuras 10, 11 y 12, se muestran estos valores para $n=1150$ y $n=1350$ para difractor circular, cuadrado y elíptico respectivamente. 

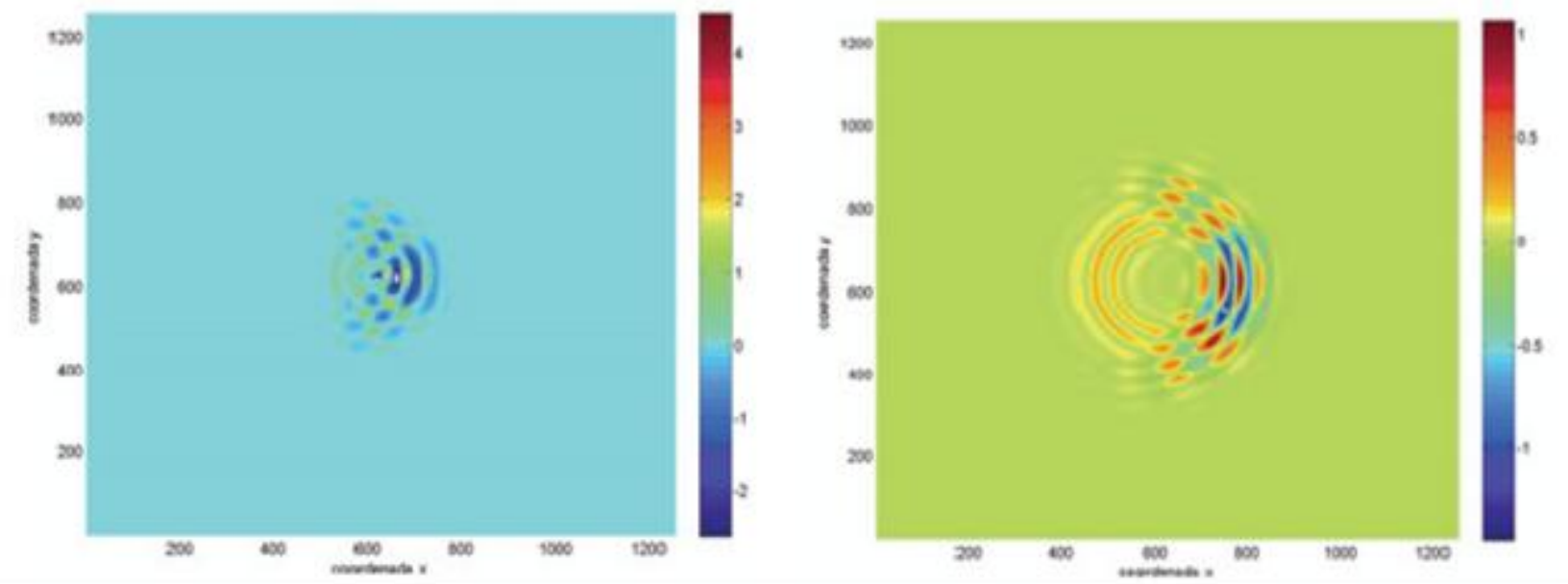

Fig. 10: Campo Eléctrico con difractor circular, a la izquierda $n=1150$, a la derecha $n=1350$
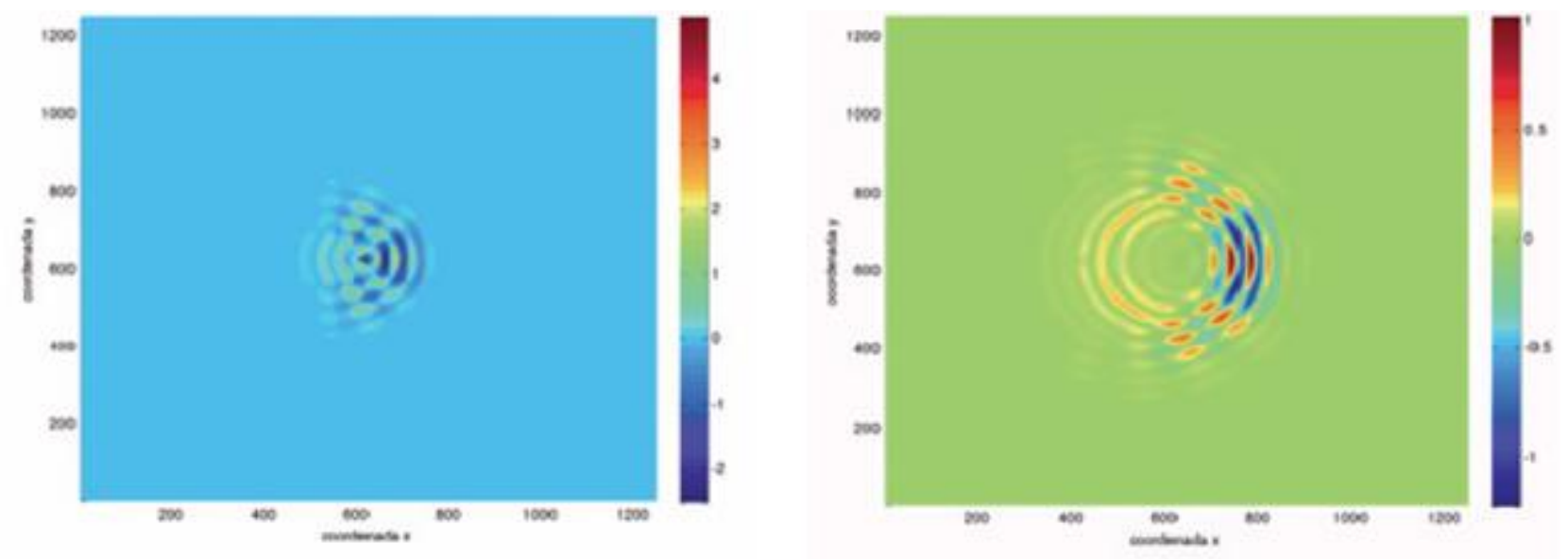

Fig. 11: Campo Eléctrico con difractor cuadrado, a la izquierda $n=1150$, a la derecha $n=1350$
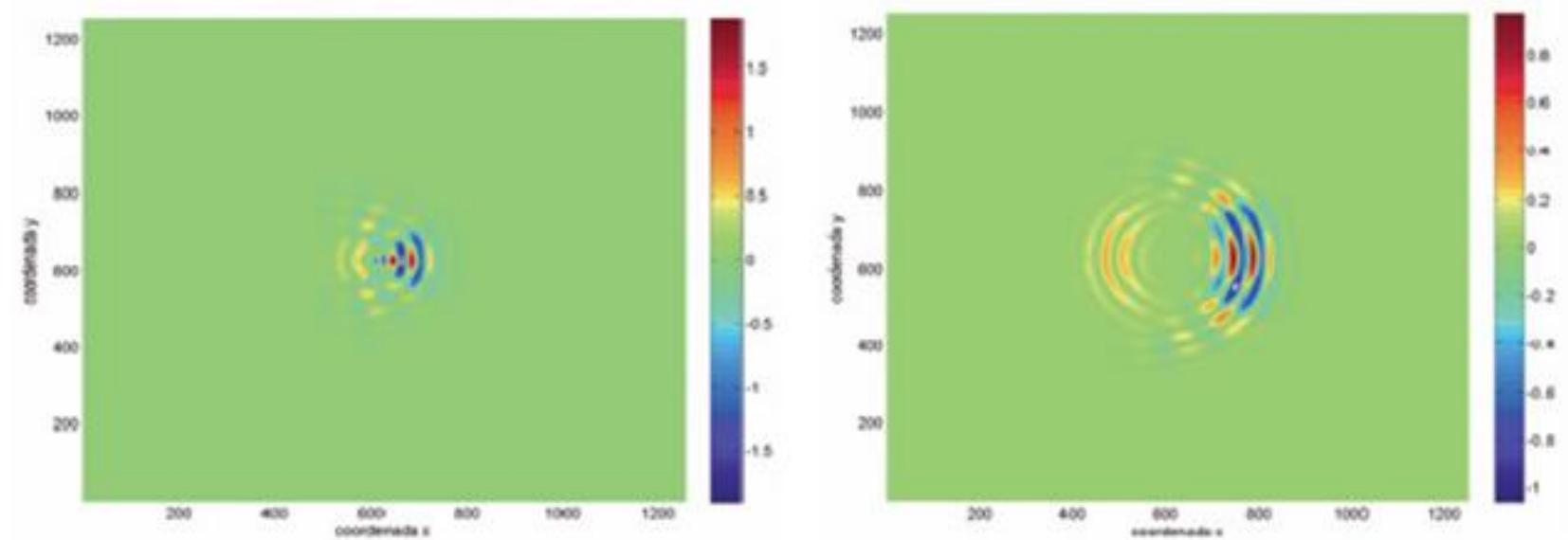

Fig. 12: Campo Eléctrico con difractor circular, a la izquierda $n=1150$, a la derecha $n=1350$

La Potencia Reflejada se obtiene en cada uno de los 15 sensores, para el caso del dieléctrico cuadrado, se logra determinar que existe simetría entre los probes P1 y P15, P2 y P14, P3 y P13, P4 y P12, P5 y P11, P6 y P10, P7 y P9, que corresponde a los resultados esperados, algunos de estos resultados se muestran en la Figura 13, en donde se presenta la comparación de la Potencia Reflejada obtenida en los sensores a) P1 y P15, b) P3 y P13 y c) P7 y P9. La simetría en las lecturas de Potencia Reflejada, es la segunda forma en que podemos validar la robustez del algoritmo de la simulación, ya que tanto la forma de onda y los valores obtenidos son exactamente los mismos, tal como se esperaba. 
P1

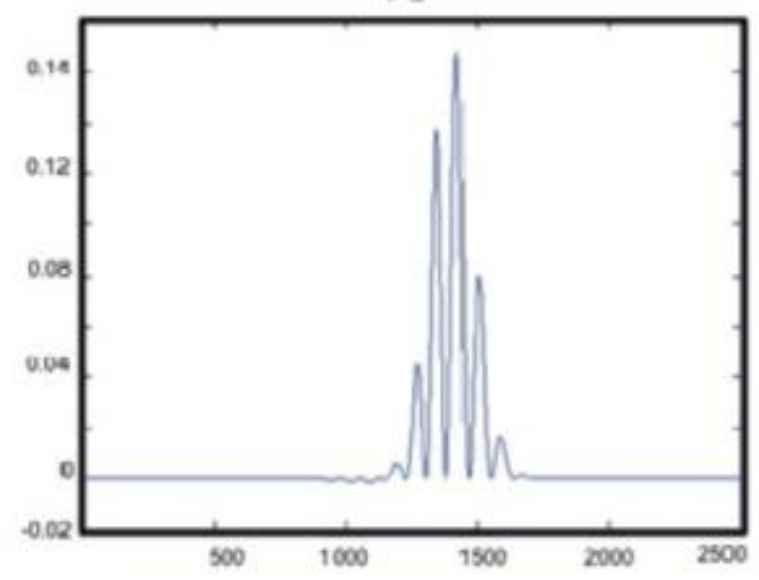

(a)

P3

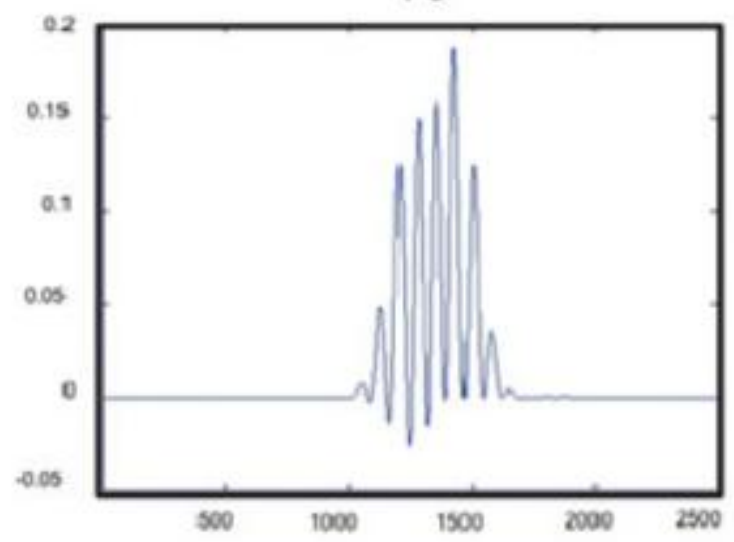

(b)

P7

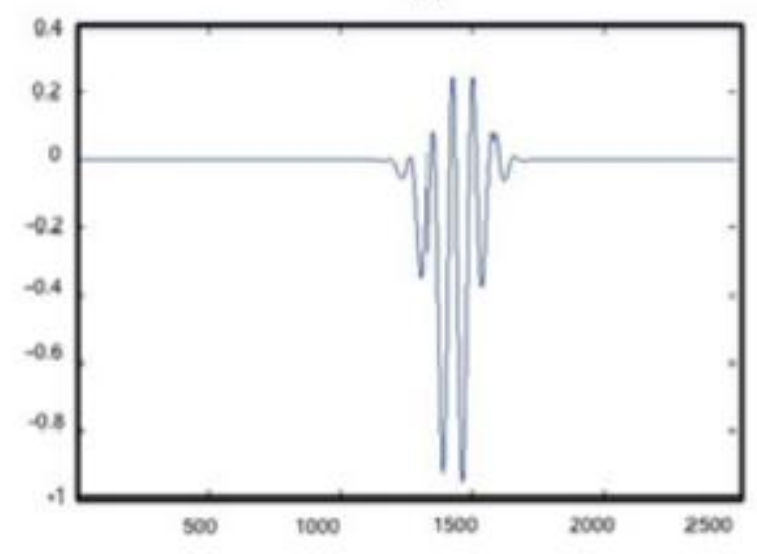

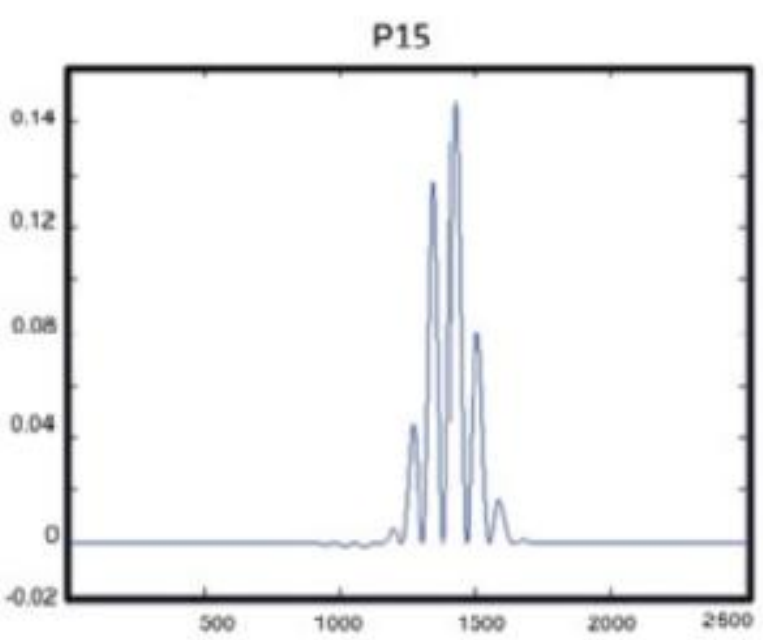

P13

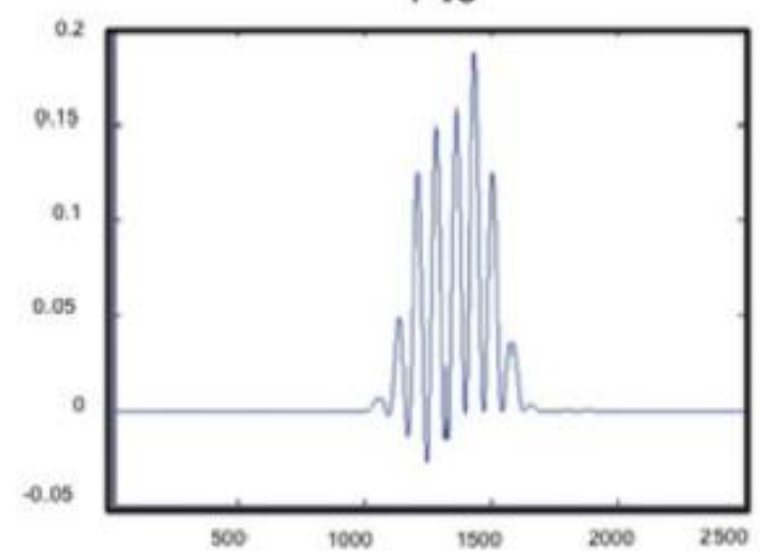

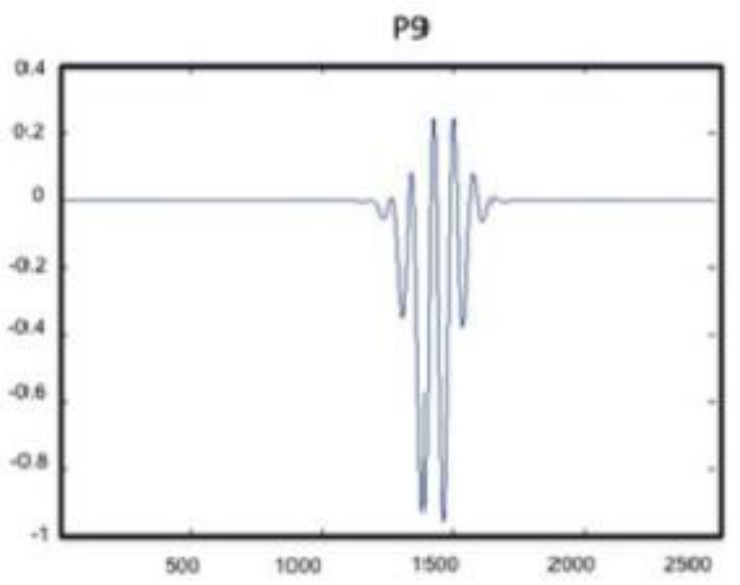

(c)

Fig. 13: Representación de valores de Potencia Reflejada (watts) obtenidos en puntos equidistantes del sistema, con respecto al espacio temporal.

\section{CONCLUSIONES}

Se ha realizado la simulación de un arreglo circular de 16 antenas, con una Antena de Transmisión (Tx1 a Tx16) y 15 Antenas Receptoras (Rx) que funcionan como sensores o probes (P1 a P15), colocadas a 22.5 de distancia entre ellas. Se presentan tres casos de estudio de acuerdo a la geometría del difractor colocado en el interior de la región de cálculo: forma circular, cuadrada y elíptica. Se hace uso de herramientas de electromagnetismo computacional como son los métodos de Diferencias Finitas en el Dominio del Tiempo, Condiciones de Frontera de Absorción y condiciones de Conductor Eléctrico Perfecto, para la simulación adecuada del sistema. Se comprueba la robustez del algoritmo al poder comprobar que 
no existen reflexiones espurias en la periferia de la región PML, así mismo que existe simetría en puntos equidistantes a la ubicación de la antena de transmisión donde se logra obtener valores de Potencia Reflejada y del Campo Eléctrico del sistema y por lo tanto, el algoritmo ya puede ser utilizado para la simulación de diversos arreglos circulares de antenas, como base del proyecto de investigación enfocado a la detección oportuna de cáncer de mama, como ha sido sugerido por varios autores mencionados en la Introducción de este artículo.

\section{REFERENCIAS}

Allen B., Dohler M., Okon E., Malik W., Brown A., Edwards D. Ultra-wideband Antennas and Propagation for Communications, Radar and Imaging, Wiley, (2007).

Amineh R.K., Ravan M.,Trehan A., Nikolova N.K., Near - field microwave imaging based on aperture raster scanning with TEM horn antennas, IEEE Transactions on Antennas and Propagation, 59(3),928-940, (2011).

Aponte G., Escobar A., Pinedo C. y Arizabaleta G. Medición de Campos Electromagnéticos en la Ciudad de Cali, Colombia, Información Tecnológica, 18(3), 39-47, (2007).

Bassi M., Bevilacqua A., Gerosa A. Neviani A., Integrate SFCW transceivers for UWB breast cancer immaging: Architectures and circuit constraints, IEEE Transactions on circuits and systems, 59(6), 12281241, (2012)

Benavides A., Alvarez M., Enciso M., Sosa J., Analysis of polynomial and geometric conductivity profiles in PML layers: a comparison, Progress in Electromagnetics Research Symposium, PIER (2010).

Benavides A., Nieto M., Enciso M., Sosa J., Parametric analysis of perfect matched layer model of finite difference time domain method, Applied Mechanics and Materials, (15), 139-144, (2009).

Benavides M., Álvarez M., Calderón C., Sosa J., Galaz M., Rodríguez M., Enciso M., Márquez C., A novel set of reduced equations to model perfect matched layer (PML) in FDTD, Revista Mexicana de Física E, 57(1), 25 - 31, (2011).

Berenger J, A perfectly matched layer for the absorption of electromagnetic waves, Journal of Computational Physics, (114), 185-200, (1994).

Conceicao R., O’ Halloran M., Glavin M., Jones E., Comparison of planar and circular antenna configurations for breast cancer detection using microwave imaging, Progress In Electromagnetics Research, PIER 99, 1(20), (2009).

Converse M., Bond E.J., Hagnes S.C., Van Veen B.D., Ultrawide-band microwave space time beamforming for hyperthermia treatment of breast cancer: A computational feasibility study, IEEE Transactions on Antennas and Propagation, 52, 1876-1889, (2004).

Converse M., Bond E.J., Hagnes S.C., Van Veen B.D. A computational study of ultra-wideband versus narrowband microwave for breast cancer treatment, 54(5), 1890-1897, (2006).

De Lorenzo M., Vera M. y Santalla del Río V., 3D Microwave breast tumor detection study of system performance, IEEE Transaction on Biomedical - Engineering, 55(12), 2772-2777, (2008).

Elsherbeni A., Demir V., The Finite-Difference Time-Domain Method for Electromagnetics with MATLAB Simulations, SciTech Publishing Inc, 187-215, Raleigh N.C, USA, (2009).

Fear E., Stuchly M., Microwave Detection of breast cancer. IEEE Transactions on Microwave Theory and Technique, 48 (11), 1854-1863, (2000).

Fear E., Li X., Hagness S. y Stuchly M., Confocal Microwave Imaging for breast cancer detection: Localization of tumors in three dimensions, IEEE Transactions Biomedical Engineering, 49(8), 812-822, (2002).

Hagness S.C, Taflove A., and Bridges J.E., Two Dimensional FDTD analysis of a pulsed microwave confocal system for breast cáncer detection: Fixed- focus and antenna-array sensors, IEEE Transactions Biomedical Engineering, 45, 1470-1479, (1998). 
Hagness S.C, Taflove A., and Bridges J.E., Three-Dimensional FDTD Analysis of a pulsed microwave confocal system for breast cancer detection: Design of an antenna- array Element, IEEE Transactions on Antennas and Propagation, 47-(5), 783-791, (1999).

Henriksson T., Joachimowicsz N. Y Bolomey J., Quantitativa Microwave Imaging for breast Cancer detection using a planar 2.45 GHz System, IEEE Transaction, 1-8, (2009).

Johnson J.E., Takenaka T., Hong Ping K.A., Honda S., y Tanaka T., Advances in the 3-D forward-BackwardTime-Stepping (FBTS) inverse scattering Technique for breast cancer detection, IEEE Transactions on Biomedical Engineering, 56(9), 2232-2243, (2009).

Li X., Davis Sh., Hagness S., Van der Weide D. y Van Veen B., Microwave Imaging via space-time beamforming experimental investigation of tumor detection in multi - layer breast phantoms, IEEE Transactions on Microwave Theory and Techniques, 52(8), 1856-1865, (2004).

Meaney P., Fanning M. Li D.,Poplack S. y Paulsen K., A CLinical Prototype for active Microwave Imaging of the breast, IEEE Transactions on Microwave Theory and Techniques, 48(11), 1841-1852, (2000).

Nilavalan R., Gibedemah A., Craddock I.J., Li X. y Hagness S.C., Numerical Investigation of breast tumour detection using multi-static radar, Electronics Letters, 39(25), (2003).

O'Halloran M., Jones E., Glavin M.,Quasi - Multistatic MIST Beamforming for the early detection of breast cancer. IEEE Transactions on Biomedical Engineering, 57(4), 830-840, (2010).

Taflove A., Hagness S., Computational Electrodynamics, the Finite-Difference Time- Domain Method, $3^{\text {a }}$ edición, 51-105, Artech House, Norwood M.A., USA.(2005).

Shea J., Kosmas P., Hagness S. y Van Veen B.,Three- dimensional microwave imaging of realistic numerical breast phantoms via a multiple-frecuency inverse scattering technique, Med. Phys, 37(8), 42104226, (2010).

Sullivan D., Electromagnetic Simulation using the FDTD Method, IEEE Press Series on RF and Microwave Technology, 51-58, New YorK USA, (2000).

Winters D.W., Shea J.D., Madsen E.L., Frank G.R., Van Veen B.D., Hagness S.C., Estimating the breast surface using UWB microwave Monostatic Backscatter Measurements. IEEE Transactions on Biomedical Engineering, 55(1), 247-256, 2008.

Xie Y., Guo B., Xu L., Li J. y Stoica P. Multistatic Adaptative Microwave Imaging for early breast cancer detection, IEEE Transaction on Biomedical- Engineering, 53(8), 1647-1657, (2006).

Yu W., Mittra R., Su T., Liu Y y Yang X. Parallel Finite- Difference Time- Domain Method. Artech House. 132, Norwood M.A., USA (2006). 